\title{
SINGULARITIES OF SYMPLECTIC AND LAGRANGIAN MEAN CURVATURE FLOWS
}

\author{
XIAOLI HAN, JIAYU LI
}

\begin{abstract}
In this paper we study the singularities of the mean curvature flow from a symplectic surface or from a Lagrangian surface in a Kähler-Einstein surface. We prove that the blow-up flow $\Sigma_{s}^{\infty}$ at a singular point $\left(X_{0}, T_{0}\right)$ of a symplectic mean curvature flow $\Sigma_{t}$ or of a Lagrangian mean curvature flow $\Sigma_{t}$ is a non trivial minimal surface in $\mathbf{R}^{4}$, if $\Sigma_{-\infty}^{\infty}$ is connected.
\end{abstract}

\section{INTRODUCTION}

Suppose that $M$ is a compact Kähler-Einstein surface. Let $\omega$ be the Kähler form on $M$ and $\langle\cdot, \cdot\rangle$ be the Kähler metric, the Kähler angle $\alpha$ of $\Sigma$ in $M$ is defined by

$$
\left.\omega\right|_{\Sigma}=\cos \alpha d \mu_{\Sigma}
$$

where $d \mu_{\Sigma}$ is the area element of $\Sigma$ of the induced metric from $\langle$,$\rangle . We call \Sigma$ a symplectic surface if $\cos \alpha>0, \Sigma$ a Lagrangian surface if $\cos \alpha=0$, and call $\Sigma$ a holomorphic cure if $\cos \alpha \equiv 1$.

It is proved in [4], [2] and [23] that, if the initial surface is symplectic, then along the mean curvature flow, at each time $t$ the surface is still symplectic, which we call a symplectic mean curvature flow. It is proved in [19] that, if the initial surface is Lagrangian, then along the mean curvature flow, at each time $t$ the surface is still Lagrangian, which we call a Lagrangian mean curvature flow.

We [8] showed that, if the scalar curvature of the Kähler-Einstein surface is positive and the initial surface is sufficiently close to a holomorphic curve, then the mean curvature flow has a global solution and it converges to a holomorphic curve.

In general, the mean curvature flows may produce singularities. The beautiful results on the nature of singularities of the mean curvature flows of convex hypersurfaces have been obtained by Huisken [11], Huisken-Sinestrari [12],[13] and White [24]. In [9] we obtain the relation between the maximum of the Kähler anlge and the maximum of $|H|^{2}$ on the blow-up flow of the symplectic mean curvature flow or the calibrated Lagrangian mean curvature flow.

It is well-known (see [9]) that, a sequence of rescaled surfaces at a singular point $\left(X_{0}, T_{0}\right)$ converges strongly to a blow-up flow $\Sigma_{s}^{\infty}$, for $s \in(-\infty, 0]$ if the singular point is of type I, for $s \in(-\infty,+\infty)$ if the singular point is of type II. In this paper, we show that $\Sigma_{s}^{\infty}$ is a non trivial holomorphic curve with finite total curvature and bounded Gauss curvature in $\mathbf{C}^{2}$, if $\Sigma_{-\infty}^{\infty}$ is connected. It is well known that the total

Key words and phrases. Symplectic surface, holomorphic curve, lagrangian surface, minimal lagrangian surface, mean curvature flow. 
curvature of a non-flat minimal surface with finite total curvature in $\mathbf{C}^{2}$ achieves only discrete values $-2 \pi N$ where $N$ is a nature number. We therefore believe that the size of the singular set can be controlled.

More precisely, let $T$ be a discrete singular time and $\left(X_{0}, T\right)$ be a singular point in $M$, one shows (see [9]) that there are sequences $r_{k} \rightarrow 0,0<\sigma_{k} \leq r_{k} / 2, t_{k} \in$ $\left[T-\left(r_{k}-\sigma_{k}\right)^{2}, T-r_{k}^{2} / 4\right], F\left(x_{k}, t_{k}\right)=X_{k} \in \bar{B}_{r_{k}-\sigma_{k}}\left(X_{0}\right)$, such that

$$
\lambda_{k}^{2}=|A|^{2}\left(X_{k}\right)=|A|^{2}\left(x_{k}, t_{k}\right)=\sup _{\left[T-\left(r_{k}-\sigma_{k}\right)^{2}, T-r_{k}^{2} / 4\right]} \sup _{\Sigma_{t} \cap B_{r_{k}-\sigma_{k}}\left(X_{0}\right)}|A|^{2},
$$

and

$$
\sup _{\left[t_{k}-\left(\sigma_{k} / 2\right)^{2}, t_{k}\right]} \sup _{\Sigma_{t} \cap B_{r_{k}-\sigma_{k} / 2}\left(X_{0}\right)}|A|^{2} \leq 4 \lambda_{k}^{2} .
$$

Choose a normal coordinates in a neighborhood of $X_{0}$, express $F$ in this coordinates, and consider the following sequence of rescaled surfaces

$$
F_{k}(x, s)=\lambda_{k}\left(F\left(x_{k}+x, t_{k}+\lambda_{k}^{-2} s\right)-F\left(x_{k}, t_{k}\right)\right), s \in\left[-\lambda_{k}^{2} \sigma_{k}^{2} / 4,0\right] .
$$

It is clear that $\left|A_{k}\right|(0,0)=1$ and $\left|A_{k}\right|^{2} \leq 4$. Denote the rescaled surfaces by $\Sigma_{s}^{k}$, then $\Sigma_{s}^{k} \rightarrow \Sigma_{s}^{\infty}$ in $C^{2}\left(B_{R}\left(X_{0}\right) \times[-R, R]\right)$ for any $R>0$ and any $B_{R}\left(X_{0}\right) \subset \mathbf{R}^{4}$. We call $\Sigma_{s}^{\infty}$ a blow-up flow.

If there exists $R_{0}>0$ such that, for all $R>R_{0}, \Sigma_{s}^{\infty} \cap B_{R}(0) \neq \emptyset$ for sufficiently large $-s$, then $\Sigma_{s}^{\infty}$ converges in $C^{2}$ to $\Sigma_{-\infty}^{\infty}$ in $B_{R}(0)$ as $s \rightarrow \infty$ for all $R>R_{0}$.

We prove the following main theorem. In fact, we prove a more general result (see Theorem 2.4) which implies the following one.

Main Theorem Let $M$ be a Kähler-Einstein surface and $\Sigma_{0}$ be a symplectic surface in $M$. If the limit $\Sigma_{-\infty}^{\infty}$ of the blow-up flow $\Sigma_{s}^{\infty}$ at infinity is connected, then $\Sigma_{s}^{\infty}$ is independent of $s$ denoted by $\Sigma^{\infty}$, and $\Sigma^{\infty}$ is a non trivial holomorphic curve in $\mathbf{C}^{2}$ with Gauss curvature $-2 \leq K \leq 0$, and finite total curvature

$$
-\int_{\Sigma^{\infty}} K d V=2 \pi N
$$

where $N$ is a nature number.

Remark Let $A_{\infty}$ be the second fundamental form of $\Sigma^{\infty}$ in $\mathbf{C}^{2}$, then $\left|A_{\infty}\right|^{2}=-2 K$, so we can also write the last identity as

$$
\int_{\Sigma^{\infty}}\left|A_{\infty}\right|^{2} d V=4 \pi N
$$

Due to the theorem, we believe that, once we control $\int_{\Sigma_{t}}|A|^{2} d \mu_{t}$, we can control the singular size of the mean curvature flow. Based on it, we propose a conjecture at end. 
Conjecture A symplectic mean curvature flow in a Kähler-Einstein surface blows up at most countable discrete times and at each blow-up time, the blow-up set consists of at most finitely many points.

We prove some similar results in the case of the Lagrangian mean curvature flows.

\section{Properties of BlOW-Up Flows of Symplectic mean CURVAtURe Flows}

In this section, we prove our main theorem.

Let $T$ be an isolated singular time, that is, the mean curvature flow exists in $t \in[T-\epsilon, T)$, and $\left(X_{0}, T\right)$ be a blow-up point. From the main theorem in [2] and [23], we know that this is a type II singularity. Recall that [9], we can define a sequence of rescaled surfaces $\Sigma_{s}^{k}$ around $\left(X_{0}, T\right)$. For each fixed $R>0$, by parabolic estimates, we have that $\Sigma_{s}^{k} \rightarrow \Sigma_{s}^{\infty}$ in $C^{2}\left(B_{R}(0) \times[-R, R]\right)$ for any $B_{R}(0) \subset \mathbf{C}^{2}$, and $\Sigma_{s}^{\infty}$ also evolves along the mean curvature flow with the property that

$$
|A|^{2}(0,0)=1 \text {, and }|A|^{2} \leq 4 .
$$

By the evolution equation derived in [2], we see that, along the mean curvature flow $\Sigma_{s}^{\infty}, \cos \alpha$ satisfies

$$
\left(\frac{\partial}{\partial t}-\Delta\right) \cos \alpha=\left|\bar{\nabla}_{0} J_{\Sigma_{s}^{\infty}}\right|^{2} \cos \alpha
$$

where $\bar{\nabla}_{0}$ is the classical derivative in $\mathbf{R}^{4}$.

By the monotonicity inequality (Proposition 3.2 in [9]), we have, for any $R>0$, $s<0$,

$$
\mu_{s}^{\infty}\left(\Sigma_{s}^{\infty} \cap B_{R}(0)\right) \leq C R^{2},
$$

where $C>0$ does not depend on $s$ or $R$. Since $M$ is compact, then there exists a constant $\delta$ such that $\cos \alpha \geq \varepsilon_{0}$ on $M$. It is easy to see that $\cos \alpha$ is scaling invariant, thus $\cos \alpha \geq \varepsilon_{0}$ on $\Sigma_{s}^{\infty}$, for all $s \in(-\infty,+\infty)$. Therefore, on $\Sigma_{s}^{\infty}$, the Isoperimetric inequality holds. That is,

Proposition 2.1. There is a positive constant $C\left(\epsilon_{0}\right)$ which depends only on $\epsilon_{0}$ such that, for any open smooth (connected) domain $A \subset \Sigma_{s}^{\infty}$,

$$
\text { Area }(A) \leq C\left(\epsilon_{0}\right)(\operatorname{Length}(\partial A))^{2} .
$$

Proof. By Theorem 30.1 in [Si1], there is an integral current $B$ with compact support such that $\partial B=\partial A$ and

$$
\operatorname{Area}(B) \leq C(\text { Length }(\partial A))^{2},
$$

where $C$ is an absolute positive constant. 
Let $T$ be the cone over $A-B$ with $\partial T=A-B$. Since $d \omega=0$ and $\left.\omega\right|_{\Sigma_{s}^{\infty}}=\cos \alpha d \mu_{s}^{\infty}$, we have

$$
\begin{aligned}
\operatorname{Area}(A) & \leq \frac{1}{\epsilon_{0}} \int_{A} \omega \\
& =\frac{1}{\epsilon_{0}} \int_{B} \omega+\partial T(\omega)=\frac{1}{\epsilon_{0}} \int_{B} \omega \\
& \leq \frac{1}{\epsilon_{0}} \operatorname{Area}(B) \\
& \leq C\left(\epsilon_{0}\right)(\operatorname{Length}(\partial A))^{2}
\end{aligned}
$$

Q. E. D.

Fix $R>0$. For any point $x$ in the connected components of $\Sigma_{s}^{\infty} \cap B_{R}(0)$ that intersect with $B_{R / 2}(0)$, denote the intrinsic ball of radius $r$ around $x$ by $\hat{B}_{r}(x)$. The isoperimetric inequality implies that

$$
\operatorname{Vol}\left(\hat{B}_{r}(x)\right) \geq C r^{2}
$$

where $C$ is a constant which depends only on $\varepsilon_{0}$. By $(2.2)$, we see that $\Sigma_{s}^{\infty} \cap B_{R}(0)$ contains at most finite many connected components which intersect with $B_{R / 2}(0)$. We denote it by $\Sigma_{s}^{(\infty, l)}, l=1, \cdots, L$.

Proposition 2.2. If the blow-up flow of a symplectic mean curvature flow is minimal, that is $H \equiv 0$, it must be holomorphic.

Proof: From [5] we know that on the minimal surface $\cos \alpha$ satisfies that,

$$
-\Delta \cos \alpha=2|\nabla \alpha|^{2} \cos \alpha=2 \frac{|\nabla \cos \alpha|^{2}}{1-\cos ^{2} \alpha} \cos \alpha
$$

at the points which are not holomorphic. Fix $R>0$, let $\phi(x) \in C^{\infty}\left(B_{2 R}(0)\right)$ be a cut-off function such that $\phi \equiv 1$ in $B_{R}(0)$ and $\phi \equiv 0$ outside of $B_{2 R}(0)$. Multiplying the equation $(2.4)$ by $\left(1-\cos ^{2} \alpha\right) \phi^{2}$ and integrating by parts, we get that,

$$
2 \int|\nabla \cos \alpha|^{2} \cos \alpha \phi^{2}=\int \sin ^{2} \alpha \phi \nabla \phi \cdot \nabla \cos \alpha .
$$

By Schwartz inequality,

$$
\int \sin ^{2} \alpha \phi \nabla \phi \cdot \nabla \cos \alpha \leq \frac{1}{2} \int \frac{\sin ^{4} \alpha}{\cos \alpha}|\nabla \phi|^{2}+\frac{1}{2} \int|\nabla \cos \alpha|^{2} \cos \alpha \phi^{2} .
$$

Plugging inequality (2.6) into (2.5), we get,

$$
\int|\nabla \cos \alpha|^{2} \cos \alpha \phi^{2} \leq \int \frac{\sin ^{4} \alpha}{\cos \alpha}|\nabla \phi|^{2}
$$

(2.2) yields,

$$
\int_{\Sigma^{\infty} \cap B_{R}(0)}|\nabla \cos \alpha|^{2} \leq C\left(\varepsilon_{0}\right) \frac{\operatorname{vol}\left(B_{2 R}\right)}{R^{2}} \leq C
$$


Let $R \rightarrow \infty$, we get that,

$$
\int_{\Sigma^{\infty}}|\nabla \cos \alpha|^{2} \leq C
$$

Multiplying equation (2.4) by $\cos ^{p} \alpha \phi^{2}$, where $p>0$ will be determined later.

$$
2 \int \cos ^{p+1} \alpha|\nabla \alpha|^{2} \phi^{2}-p \int \cos ^{p-1} \alpha|\nabla \cos \alpha|^{2} \phi^{2}=2 \int \cos ^{p} \alpha \phi \nabla \phi \cdot \nabla \cos \alpha .
$$

Using Holder inequality , (2.7), and (2.2), we have

$$
\begin{aligned}
2 \int \cos ^{p} \alpha \phi \nabla \phi \nabla \cos \alpha & \leq 2\left(\int \cos ^{2 p} \alpha|\nabla \phi|^{2} \phi^{2}\right)^{1 / 2}\left(\int_{B_{2 R} / B_{R}}|\nabla \cos \alpha|^{2}\right)^{1 / 2} \\
& \leq C\left(\int_{B_{2 R} / B_{R}}|\nabla \cos \alpha|^{2}\right)^{1 / 2} \rightarrow 0, \text { as } R \rightarrow \infty
\end{aligned}
$$

Thus we have,

$$
\int\left(2 \cos ^{2} \alpha-p \sin ^{2} \alpha\right)|\nabla \alpha|^{2} \cos ^{p-1} \alpha=0 .
$$

Choosing $p$ such that $\frac{p}{p+2}<\varepsilon_{0}^{2}$, then $2 \cos ^{2} \alpha-p \sin ^{2} \alpha>c\left(\varepsilon_{0}\right)>0$, thus

$$
\int|\nabla \alpha|^{2} \cos ^{p-1} \alpha=0
$$

which implies that $|\nabla \alpha|^{2}=0$. Therefore, $\Sigma$ is holomorphic with some complex structure in $\mathbf{C}^{2}$.

Q. E. D.

Remark 2.3. This result can also be deduced from the theorem in [6].

It is clear that, if $\Sigma_{s}^{(\infty, l)} \cap B_{R}(0) \neq \emptyset$ for all $-s$ sufficiently large, then $\Sigma_{s}^{(\infty, l)}$ converges in $C^{2}\left(B_{R}(0)\right)$ to $\Sigma_{-\infty}^{(\infty, l)}$ as $s \rightarrow-\infty$, for any $R>0$.

Definition 2.1. The component $\Sigma_{s}^{(\infty, l)}$ is called simple, if $\Sigma_{-\infty}^{(\infty, l)}$ is connected.

Theorem 2.4. Let $M$ be a Kähler-Einstein surface and $\Sigma_{0}$ be a symplectic surface in M. Each simple connected component $\Sigma_{s}^{(\infty, l)}$ of the blow-up flow $\Sigma_{s}^{\infty}$ is independent of $s$ denoted by $\Sigma^{(\infty, l)}$, and $\Sigma^{(\infty, l)}$ is a holomorphic curve in $\mathbf{C}^{2}$ with Gauss curvature $-2 \leq K \leq 0$, and finite total curvature

$$
-\int_{\Sigma^{\infty}} K d V=2 \pi N
$$

where $N$ is a nature number.

Proof: For simplicity, we denote the simple connected component by $\Sigma_{s}^{\infty}$. The following monotonicity formula for $\Sigma_{s}^{\infty}$ is derived in [9].

Let $H\left(X, X_{0}, t, t_{0}\right)$ be the backward heat kernel on $\mathbf{R}^{4}$. Define

$$
\rho\left(X, X_{0}, t, t_{0}\right)=4 \pi\left(t_{0}-t\right) H\left(X, X_{0}, t, t_{0}\right)=\frac{1}{4 \pi\left(t_{0}-t\right)} \exp \left(-\frac{\left|X-X_{0}\right|^{2}}{4\left(t_{0}-t\right)}\right)
$$


for $t<t_{0}$. We have, for $-\infty<s<s_{0}$,

$$
\begin{aligned}
& \frac{\partial}{\partial s}\left(\int_{\Sigma_{s}^{\infty}} \frac{1}{\cos \alpha} \rho\left(F_{\infty}, 0, s, s_{0}\right) d \mu_{s}^{\infty}\right) \\
& =-\left(\int_{\Sigma_{s}^{\infty}} \frac{1}{\cos \alpha} \phi \rho\left(F_{\infty}, 0, s, s_{0}\right)\left|H_{\infty}+\frac{\left(F_{\infty}\right)^{\perp}}{2\left(s_{0}-s\right)}\right|^{2} d \mu_{s}^{\infty}\right. \\
& +\int_{\Sigma_{s}^{\infty}} \frac{1}{2 \cos \alpha} \phi \rho\left(F_{\infty}, 0, s, s_{0}\right)\left|\bar{\nabla} J_{\Sigma_{s}^{\infty}}\right|^{2} d \mu_{s}^{\infty} \\
& \left.+\int_{\Sigma_{s}^{\infty}} \frac{2}{\cos ^{3} \alpha}|\nabla \cos \alpha|^{2} \phi \rho\left(F_{\infty}, 0, s, s_{0}\right) d \mu_{s}^{\infty}\right) .
\end{aligned}
$$

Choosing $s_{0}=0, s_{1}=4 t, s_{2}=2 t$, for $t<0$ we have,

$$
\begin{aligned}
& \int_{\Sigma_{4 t}^{\infty}} \frac{1}{\cos (x, 4 t)} \frac{1}{-4 t} e^{-\frac{\left|F_{\infty}\right|^{2}}{-4 t}} d \mu_{4 t}^{\infty}-\int_{\Sigma_{2 t}^{\infty}} \frac{1}{\cos (x, 2 t)} \frac{1}{-2 t} e^{-\frac{\left|F_{\infty}\right|^{2}}{-2 t}} d \mu_{2 t}^{\infty} \\
& \geq \int_{4 t}^{2 t} \int_{\Sigma_{s}^{\infty}} \frac{1}{\cos \alpha} \rho\left(F_{\infty}, 0, s, 0\right)\left|\bar{\nabla} J_{\Sigma_{s}^{\infty}}\right|^{2} d \mu_{s}^{\infty} d s .
\end{aligned}
$$

Since $\int_{\Sigma_{s}^{\infty}} \frac{1}{\cos \alpha} \rho\left(F_{\infty}, 0, s, s_{0}\right) d \mu_{s}^{\infty}$ is uniformly bounded above (See [9]), so the left side of the above inequality tends to zero as $t \rightarrow-\infty$. Moreover,

$$
\begin{aligned}
& \int_{4 t}^{2 t} \int_{\Sigma_{s}^{\infty}} \frac{1}{\cos \alpha} \frac{1}{-s} e^{-\frac{\left|F_{\infty}\right|^{2}}{-s}}\left|\bar{\nabla} J_{\Sigma_{s}^{\infty}}\right|^{2} d \mu_{s}^{\infty} \\
& =-2 t \int_{\Sigma_{s^{\prime}}^{\infty}} \frac{1}{\cos \alpha} \frac{1}{-s^{\prime}} e^{-\frac{\left|F_{\infty}\right|^{2}}{-s^{\prime}}}\left|\bar{\nabla} J_{\Sigma_{s^{\prime}}}\right|^{2} d \mu_{s^{\prime}}^{\infty} \\
& \geq C \int_{\Sigma_{s^{\prime}}^{\infty}}\left|\bar{\nabla} J_{\Sigma_{s^{\prime}}^{\infty}}\right|^{2} e^{-\frac{\left|F_{\infty}\right|^{2}}{-s^{\prime}}} d \mu_{s^{\prime}}^{\infty}
\end{aligned}
$$

where $s^{\prime} \in[4 t, 2 t], C$ is independent of $t$.

It is clear that, for any $R>0$, if $\Sigma_{s}^{\infty} \cap B_{R}(0) \neq \emptyset$ for sufficiently large $-s$, then $\Sigma_{s}^{\infty} \cap B_{R}(0)$ converges strongly to $\Sigma_{-\infty}^{\infty} \cap B_{R}(0)$ as $s \rightarrow-\infty$. By the assumption, we know that $\Sigma_{-\infty}^{\infty} \cap B_{R}(0)$ is connected for $R$ large enough, so there is $S_{0}$ such that $s<S_{0}, \Sigma_{s}^{\infty} \cap B_{R}(0)$ is connected.

Letting $t \rightarrow-\infty$, we get that on $\Sigma_{-\infty}^{\infty}$,

$$
\left|\bar{\nabla} J_{\Sigma_{-\infty}^{\infty}}\right|^{2}=0, \quad \text { that is, }|\nabla \cos \alpha|^{2}=0 .
$$

It follows that $\cos \alpha \equiv \theta_{0}, \theta_{0}$ is constant on $\Sigma_{-\infty}^{\infty}$.

We can choose the suitable complex structure on $\mathbf{R}^{4}$ such that with respect to this complex structure we have $\cos \alpha \equiv 1$ on $\Sigma_{-\infty}^{\infty}$. In fact, assume that $\omega$ is written as $\omega=d z_{1} \wedge d \bar{z}_{1}+d z_{2} \wedge d \bar{z}_{2}$ under the standard complex structure of $\mathbf{R}^{4}$. We define a new complex structure $J^{*}$ of $\mathbf{R}^{4}$ as follows:

$$
\begin{aligned}
& J^{*}\left(\partial / \partial x_{1}\right)=\theta_{0}\left(\partial / \partial y_{1}\right), J^{*}\left(\partial / \partial y_{1}\right)=-1 / \theta_{0}\left(\partial / \partial x_{1}\right) \\
& J^{*}\left(\partial / \partial x_{2}\right)=1 / \theta_{0}\left(\partial / \partial y_{2}\right), J^{*}\left(\partial / \partial y_{2}\right)=-\theta_{0}\left(\partial / \partial x_{2}\right) .
\end{aligned}
$$


Under the complex structure $J^{*}$, the complex coordinates are $z_{1}^{*}=x_{1}+\sqrt{-1} \theta_{0}^{-1} y_{1}$, $z_{2}^{*}=\theta_{0}^{-1} x_{2}+\sqrt{-1} y_{2}$. Thus $\omega^{*}=d z_{1}^{*} \wedge d \bar{z}_{1}^{*}+d z_{2}^{*} \wedge d \bar{z}_{2}^{*}$ satisfies $\left.\omega^{*}\right|_{\Sigma_{-\infty, l}^{\infty}}=d \mu_{-\infty, l}^{\infty}$, In other words, $\theta_{0} \equiv 1$.

Recall that, on $\Sigma_{s}^{\infty}, \cos \alpha$ satisfies

$$
\left(\frac{\partial}{\partial s}-\Delta\right) \cos \alpha=\left|\bar{\nabla}_{0} J_{\Sigma_{s}^{\infty}}\right|^{2} \cos \alpha
$$

Using the maximum principle on $\Sigma_{s}^{\infty}$ (See [7]), for all $s \leq S_{0}$, we can see that $\min _{\Sigma_{s}^{\infty}} \cos \alpha$ is a nondecreasing function of $s$, so $\cos \alpha \equiv 1$ on $\Sigma_{s}^{\infty}$, for all $s \leq S_{0}$. We therefore have that $\left|\bar{\nabla}_{0} J_{\Sigma_{s}^{\infty}}\right| \equiv 0$, hence $H_{s}^{\infty} \equiv 0$, and consequently, $\Sigma_{s}^{\infty}, s \leq S_{0}$, does not depend on $s$ and is a holomorphic curve. We continue this process and claim that $\Sigma_{s}^{\infty}$ is a holomorphic curve for all $s<0$.

By (2.1), we see that the second fundamental form $A_{\infty}$ of $\Sigma^{\infty}$ in $\mathbf{R}^{4}$ satisfies

$$
\left|A_{\infty}(0)\right|=1 \text { and }\left|A_{\infty}\right|^{2} \leq 4 \text {. }
$$

Let $K$ be the Gauss curvature of $\Sigma^{\infty}, R$ the curvature operator of $M$. By Gauss equation,

$$
K_{1212}=R_{1212}+\left(h_{11}^{\alpha} h_{22}^{\alpha}-h_{12}^{\alpha} h_{12}^{\alpha}\right)
$$

we get

$$
|A|^{2}=|H|^{2}-2 K_{1212}+2 R_{1212}
$$

Thus we have

$$
K=-\frac{1}{2}\left|A_{\infty}\right|^{2}
$$

We will show in the following proposition that, the total curvature of $\Sigma^{\infty}$ is finite.

Proposition 2.5. The minimal surface $\Sigma^{\infty}$ in the previous theorem is of finite type, that is, its total curvature is finite.

Proof. Since

$$
-\int_{\Sigma^{\infty}} K d \mu^{\infty}=\frac{1}{2} \int_{\Sigma^{\infty}}\left|A_{\infty}\right|^{2} d \mu^{\infty}
$$

so it suffices to prove that

$$
\int_{\Sigma^{\infty}}\left|A_{\infty}\right|^{2} d \mu^{\infty}<\infty
$$

It follows from the integral curvature estimate (Theorem 4, in [Il]) that for any $r>0$,

$$
r^{-2} \int_{t-r^{2}}^{t} \int_{\Sigma_{t} \cap B_{r}(x)}|A|^{2} d \mu_{t} d t \leq C
$$

Here $C$ does not depend on $r$. Thus as $k$ sufficiently large we have

$$
\lambda_{k}^{2} R^{-2} \int_{t_{k}-\lambda_{k}^{-2} R^{2}}^{t_{k}} \int_{\Sigma_{t} \cap B_{\lambda_{k}^{-1} R}\left(X_{k}\right)}|A|^{2} d \mu_{t} d t \leq C .
$$

This implies that

$$
R^{-2} \int_{-R^{2}}^{0} \int_{\sum_{s}^{k} \cap B_{R}(0)}\left|A_{k}\right|^{2} d \mu_{s}^{k} d s \leq C
$$


Letting $k \rightarrow \infty$, we get

$$
R^{-2} \int_{-R^{2}}^{0} \int_{\Sigma_{s}^{\infty} \cap B_{R}(0)}\left|A_{\infty}\right|^{2} d \mu_{s}^{\infty} d s \leq C .
$$

Note that $\Sigma_{s}^{\infty}$ does not depend on $s$, we have

$$
\int_{\Sigma \infty \cap B_{R}(0)}\left|A_{\infty}\right|^{2} d \mu^{\infty} \leq C
$$

Letting $R \rightarrow \infty$, we get that

$$
\int_{\Sigma^{\infty}}\left|A_{\infty}\right|^{2} d \mu^{\infty} \leq C
$$

This proves the proposition.

By Proposition 6.1 in [14], we know that

Q. E. D.

$$
-\int_{\Sigma^{\infty}} K d V=2 \pi N
$$

where $N$ is a natural number.

This proves the theorem.

Q. E. D.

Corollary 2.6. Let $M$ be a Kähler-Einstein surface and $\Sigma_{0}$ be a symplectic surface in $M$. If the blow-up flow $\Sigma_{s}^{\infty}$ contains only one connected component, and its limit at $-\infty, \Sigma_{-\infty}^{\infty}$ is connected, then $\Sigma_{s}^{\infty}$ is independent of $s$ denoted by $\Sigma^{\infty}$, and $\Sigma^{\infty}$ is a non trivial holomorphic curve in $\mathbf{C}^{2}$ with Gauss curvature $-2 \leq K \leq 0$, and finite total curvature

$$
-\int_{\Sigma^{\infty}} K d V=2 \pi N
$$

where $N$ is a nature number.

There are many works on minimal surfaces with finite total curvature, we state one of them (c.f. Theorem 6.1 in [18]), so that the readers are aware of the properties of $\Sigma^{\infty}$.

Theorem 2.7. Let $x: \Sigma \rightarrow \mathbb{R}^{m}$ be a non-flat complete minimal surface in $\mathbb{R}^{m}$. Then, the following conditions are equivalent.

(i) $M$ has finite total curvature.

(ii) $M$ is biholomorphic with a compact Riemann surface $\bar{M}$ with finitely many points removed and each $\omega_{i}=\partial x_{i}$ extends to $\bar{M}$ as a meromorphic form.

(iii) $M$ is biholomorphic with an open subset of a compact Riemann surface $\bar{M}$ and the Gauss map $G: M \rightarrow P^{m-1}(C)$ extends to a holomorphic map of $\bar{M}$ into $P^{m-1}(C)$.

It is certainly important to know when a connected component $\Sigma_{s}^{(\infty, l)}$ of the blowup flow $\Sigma_{s}^{\infty}$ is simple. Let $\rho_{s}$ be the induced distance on $\Sigma_{s}^{(\infty, l)}$ for each $s,\|\cdot\|$ be the distance in the Euclidean metric $R^{4}$, it is obvious that,

$$
\|x-y\| \leq \rho_{s}(x, y), \text { for } x, y \in \Sigma_{s}^{(\infty, l)} .
$$

If there is $C>0$ independent of $s$ such that

$$
\rho_{s}(x, y) \leq C\|x-y\|, \text { for } x, y \in \Sigma_{s}^{(\infty, l)},
$$


then $\Sigma_{s}^{(\infty, l)}$ must be simple, that is, $\Sigma_{-\infty}^{(\infty, l)}$ is connected.

We conjecture that, every connected component of a blow-up flow of a symplectic mean curvature flow is simple.

\section{Blow up ANALYSis AT INFINITY}

In this section we assume that the symplectic mean curvature flow exists for long time, then we study the structure of the singularity at infinity.

Suppose that $X_{0}$ is a blow up point at infinite, then for arbitrary sequences $t_{k} \rightarrow \infty$ the quantity

$$
\max _{\sigma \in(0, r / 2]} \sigma^{2} \sup _{\left[t_{k}-(r-\sigma)^{2}, t_{k}\right]} \sup _{\Sigma_{t} \cap B_{r-\sigma}\left(X_{0}\right)}|A|^{2} \rightarrow \infty
$$

where $r$ is a constant which is less than injective radius of $M$ at $X_{0}$. In fact,

$$
\max _{\sigma \in(0, r / 2]} \sigma^{2} \sup _{\left[t_{k}-(r-\sigma)^{2}, t_{k}\right]} \sup _{\Sigma_{t} \cap B_{r-\sigma}\left(X_{0}\right)}|A|^{2} \geq(r / 2)^{2} \sup _{\Sigma_{t_{k}} \cap B_{r / 2}\left(X_{0}\right)}|A|^{2} .
$$

It is clear that the right hand side term of the above inequality tends to infinity as $t_{k} \rightarrow \infty$.

We choose $\sigma_{k} \in(0, r / 2]$ such that

$$
\sigma_{k}^{2} \sup _{\left[t_{k}-\left(r-\sigma_{k}\right)^{2}, t_{k}\right]} \sup _{\Sigma_{t} \cap B_{r-\sigma_{k}}\left(X_{0}\right)}|A|^{2}=\max _{\sigma \in(0, r / 2]} \sigma^{2} \sup _{\left[t_{k}-(r-\sigma)^{2}, t_{k}\right] \Sigma_{t} \cap B_{r-\sigma}\left(X_{0}\right)}|A|^{2} .
$$

Let $\tilde{t}_{k} \in\left[t_{k}-\left(r-\sigma_{k}\right)^{2}, t_{k}\right], F\left(x_{k}, \tilde{t}_{k}\right)=X_{k} \in \bar{B}_{r-\sigma_{k}}\left(X_{0}\right)$, such that

$$
\lambda_{k}^{2}=|A|^{2}\left(X_{k}\right)=|A|^{2}\left(x_{k}, \tilde{t}_{k}\right)=\sup _{\left[t_{k}-\left(r-\sigma_{k}\right)^{2}, t_{k}\right]} \sup _{\Sigma_{t} \cap B_{r-\sigma_{k}}\left(X_{0}\right)}|A|^{2} .
$$

From equation (3.1) we know that $\lambda_{k}^{2} \sigma_{k}^{2} \rightarrow \infty$ as $k \rightarrow \infty$. In particular,

$$
\sup _{\left[t_{k}-\left(r-\sigma_{k} / 2\right)^{2}, t_{k}\right]} \sup _{\Sigma_{t} \cap B_{r-\sigma_{k} / 2}\left(X_{0}\right)}|A|^{2} \leq 4 \lambda_{k}^{2}
$$

and hence

$$
\sup _{\left[\tilde{t}_{k}-\left(\sigma_{k} / 2\right)^{2}, \tilde{t}_{k}\right] \Sigma_{t} \cap B_{r-\sigma_{k} / 2}\left(X_{0}\right)}|A|^{2} \leq 4 \lambda_{k}^{2} .
$$

Therefore we can consider the rescaled sequence,

$$
F_{k}(x, s)=\lambda_{k}\left(F\left(x_{k}+x, \tilde{t}_{k}+\lambda_{k}^{-2} s\right)-F\left(x_{k}, \tilde{t}_{k}\right)\right), s \in\left[-\lambda_{k}^{2} \sigma_{k}^{2} / 4,0\right] .
$$

We denote the rescaled surface by $\Sigma_{s}^{k}=F_{k}(\Sigma, s)$. By the same analysis as the one used at a finite time singularity (see [9]), we can show that $\Sigma_{s}^{k} \rightarrow \Sigma_{s}^{\infty}$ in $C^{2}\left(B_{R}(0) \times[-R, R]\right)$ for any $R>0$ and $\Sigma_{s}^{\infty}$ is a mean curvature flow in $\mathbf{C}^{2}$ which we call a blow-up flow. It is clear that the blow-up flow also satisfies the identity and inequalities (2.1) and $(2.2)$.

Similarly, we can prove the following theorem.

Theorem 3.1. Let $M$ be a Kähler-Einstein surface and $\Sigma_{0}$ be a symplectic surface in $M$. Assume that the mean curvature flow exists globally. Each simple connected component $\Sigma_{s}^{(\infty, l)}$ of the blow-up flow $\Sigma_{s}^{\infty}$ at infinity is independent of $s$ denoted by 
$\Sigma^{(\infty, l)}$, and $\Sigma^{(\infty, l)}$ is a holomorphic curve in $\mathbf{C}^{2}$ with Gauss curvature $-2 \leq K \leq 0$, and finite total curvature

$$
-\int_{\Sigma^{\infty}} K d V=2 \pi N
$$

where $N$ is a nature number.

\section{Singularities of Lagrangian mean CuRvature Flows}

In the case of Lagrangian mean curvature flow, suppose that $M$ is a Kähler-Einstein surface with scalar curvature $\mathcal{R}$ and the mean curvature form of the initial surface $\Sigma_{0}$ is exact, Smoczyk [21] showed that there exists a function $\beta$ such that

$$
\begin{aligned}
d \beta & =H \\
\frac{d \beta}{d t} & =\Delta \beta+\frac{\mathcal{R}}{4} \beta
\end{aligned}
$$

and $\beta$ is called the Lagrangian angle. Let $\tilde{\beta}=e^{-\frac{\mathcal{R}}{4} t} \beta$, we have,

$$
\frac{\partial \cos \tilde{\beta}}{\partial t}=\Delta \cos \tilde{\beta}+e^{-\frac{\mathcal{R}}{2} t}|H|^{2} \cos \tilde{\beta}
$$

It is certainly more interesting when $\mathcal{R}=0$, that is, $M$ is a Calabi-Yau surface.

Let $M$ be a compact Calabi-Yau surface with a parallel holomorphic $(2,0)$-form $\Omega$ of unit length. Then we have

$$
\left.\Omega\right|_{\Sigma_{t}}=e^{i \beta} d \mu_{t}=\cos \beta d \mu_{t}+i \sin \beta d \mu_{t}
$$

If $\cos \beta>0$, we say that $\Sigma_{t}$ is almost calibrated. Smoczyk [21] (also see [22]) showed that, if $\Sigma_{0}$ is almost calibrated, then $\Sigma_{t}$ is too, which we call an almost calibrated Lagrangian mean curvature flow. It is proved in [3] and [23] that, along an almost calibrated Lagrangian mean curvature flow, there is no Type I singularity.

Let $T$ be an isolated singular time, that is, the mean curvature flow exists in $t \in[T-\epsilon, T)$, and $\left(X_{0}, T\right)$ be a blow-up point. We consider the strong convergence of the rescaled surfaces $\Sigma_{s}^{k}$ in $B_{R}(0)$ around the singular point $X_{0}$ (c.f. [2], [9]), we have a blow-up flow $\Sigma_{s}^{\infty}$ in $\mathbb{C}^{2}$ with the Euclidean metric, which is defined on $(-\infty, 0]$ for Type I singularity and defined on $(-\infty, \infty)$ for Type II singularity. The blow-up flow satisfies the identity and the inequalities (2.1) and (2.2).

Theorem 4.1. Let $M$ be a compact Kähler surface and the initial surface $\Sigma_{0}$ be an exact Lagrangian surface with $\beta$ bounded. Each simple connected component $\Sigma_{s}^{(\infty, l)}$ of the blow-up flow $\Sigma_{s}^{\infty}$ is independent of $s$ denoted by $\Sigma^{(\infty, l)}$, and $\Sigma^{(\infty, l)}$ is a minimal surface in $\mathbf{R}^{4}$ with Gauss curvature $-2 \leq K \leq 0$, and finite total curvature

$$
-\int_{\Sigma^{\infty}} K d V=2 \pi N
$$

where $N$ is a nature number.

Proof. Recall that, by the blow-up technique, we can get a sequence of rescaled surfaces $\Sigma_{s}^{k}$ around $\left(X_{0}, T\right)$, which converges to $\Sigma_{s}^{\infty}$ strongly in $C^{2}\left(B_{R}(0) \times[-R, R]\right)$ 
for any $R>0$ and any $B_{R}(0) \subset \mathbf{R}^{4}$, with $\left|A_{k}(0)\right|=1$ and $\left|A_{k}\right|^{2} \leq 4$. Moreover, $\Sigma_{s}^{\infty}$ evolves along the mean curvature flow. Since

$$
\left(\frac{\partial}{\partial s}-\Delta\right) \beta_{s}^{k}=\frac{\mathcal{R}}{4 \lambda_{k}^{2}} \beta_{s}^{k},
$$

we can see that, along the mean curvature flow $\Sigma_{s}^{\infty}, \beta$ satisfies

$$
\left(\frac{\partial}{\partial s}-\Delta\right) \beta=0
$$

In the following, we denote the simple connected component $\Sigma_{s}^{(\infty, l)}$ of the blow-up flow simply by $\Sigma_{s}^{\infty}$.

One can show the monotonicity formula (c.f. [3], [9], [16],[17])

$$
\begin{aligned}
& \frac{\partial}{\partial s}\left(\int_{\Sigma_{s}^{\infty}} \beta^{2} \rho\left(F_{\infty}, 0, s, s_{0}\right) d \mu_{s}^{\infty}\right) \\
& \leq-\left(\int_{\Sigma_{s}^{\infty}} \beta^{2} \rho\left(F_{\infty}, 0, s, s_{0}\right)\left|H_{\infty}+\frac{\left(F_{\infty}\right)^{\perp}}{2\left(s_{0}-s\right)}\right|^{2} d \mu_{s}^{\infty}\right. \\
& \left.+\int_{\Sigma_{s}^{\infty}}|\nabla \beta|^{2} \rho\left(F_{\infty}, 0, s, s_{0}\right) d \mu_{s}^{\infty}\right) .
\end{aligned}
$$

From this monotonicity formula, similarly we can get that on $\Sigma_{-\infty}^{\infty}$,

$$
|\nabla \beta|=0,
$$

so, $\beta \equiv$ constant on $\Sigma_{-\infty}^{\infty}$, it can be normalized to be zero. By the evolution equation (4.2), we see that $\beta \equiv 0$ on $\Sigma_{s}^{\infty}$. Since $H_{\Sigma_{s}^{\infty}}=d \beta$, we have that $H_{\Sigma_{s}^{\infty}} \equiv 0$, and consequently, $\Sigma_{s}^{\infty}$ does not depend on $s$ and is a minimal surface in $\mathbb{R}^{4}$. Because $\Sigma_{s}^{k}$ converges to $\Sigma^{\infty}$ in $C^{2}\left(B_{R}(0)\right)$ for any $R>0$ as $k \rightarrow \infty$, we see that $\Sigma^{\infty}$ is a complete minimal surface. Let $K$ be the Gauss curvature of $\Sigma^{\infty}$, similarly we can get that

$$
\int_{\Sigma \infty}\left|A_{\infty}\right|^{2} d \mu^{\infty} \leq C
$$

by Proposition 6.1 in [14], we see that that

$$
-\int_{\Sigma^{\infty}} K d V=2 \pi N
$$

where $N$ is a nature number. This completes the proof of the theorem.

Q. E. D.

Corollary 4.2. Let $M$ be a compact Kähler surface and the initial surface $\Sigma_{0}$ be an exact Lagrangian surface with $\beta$ bounded. If the blow-up flow $\Sigma_{s}^{\infty}$ contains only one connected component, and its limit at $-\infty, \Sigma_{-\infty}^{\infty}$ is connected, then $\Sigma_{s}^{\infty}$ is independent of $s$ denoted by $\Sigma^{\infty}$, and $\Sigma^{\infty}$ is a non trivial minimal surface in $\mathbf{R}^{4}$ with Gauss curvature $-2 \leq K \leq 0$, and finite total curvature

$$
-\int_{\Sigma^{\infty}} K d V=2 \pi N
$$

where $N$ is a nature number. 
By similar arguments, we can show the following theorem. We omit the proof here.

Theorem 4.3. Let $M$ be a compact Kähler surface and the initial surface $\Sigma_{0}$ be an exact Lagrangian surface with $\beta$ bounded. Assume that the mean curvature flow has a long time solution. Each simple connected component $\Sigma_{s}^{(\infty, l)}$ of the blow-up flow $\Sigma_{s}^{\infty}$ at infinity is independent of $s$ denoted by $\Sigma^{(\infty, l)}$, and $\Sigma^{(\infty, l)}$ is a minimal surface in $\mathbf{R}^{4}$ with Gauss curvature $-2 \leq K \leq 0$, and finite total curvature

$$
-\int_{\Sigma^{\infty}} K d V=2 \pi N
$$

where $N$ is a nature number.

\section{REFERENCES}

[1] K. Brakke, The motion of a surface by its mean curvature, Princeton Univ. Press, 1978.

[2] J. Chen and J. Li, Mean curvature flow of surface in 4-manifolds, Adv. Math., 163 (2001), 287-309.

[3] J. Chen and J. Li, Singularity of mean curvature flow of Lagrangian submanifolds, Invent. Math., 156 (2004), 25-51.

[4] J. Chen and G. Tian, Minimal surfaces in Riemannian 4-manifolds, Geom. Funct. Anal., 7 (1997), 873-916.

[5] S. S. Chern and J. Wolfson, Minimal surfaces by moving frames, Amer. J. Math., 105 (1983), 59-83.

[6] S. Y. Cheng, S. T. Yau, Differential equations on Riemannian manifolds and their geometric applications, Comm. Pure Appl. Math., 28 (1975), no. 3, 333-354.

[7] K. Ecker and G. Huisken, Mean curvature evolution of entire graphs, Ann. Math., 130 (1989), 453-471.

[8] X. Han and J. Li, The mean curvature flow approach to the symplectic isotopy problem, IMRN, 26 (2005), 1611-1620.

[9] X. Han and J. Li, The second type singularity of symplectic and Lagrangian mean curvature flows, Preprint.

[10] G. Huisken, Asymptotic behavior for singularities of the mean curvature flow, J. Diff. Geom. 31 (1990), 285-299.

[11] G. Huisken, Contracting convex hypersurfaces in Riemannian manifolds by their mean curvature, Invent. math., 84 (1986), 463-480.

[12] G. Huisken and C. Sinestrari, Mean curvature flow singularities for mean convex surfaces, Calc. Var. PDE 8 (1999), 1-14.

[13] G. Huisken and C. Sinestrari, Convexity estimates for mean curvature flow and singularities of mean convex surfaces, Acta Math., 183 (1999), 45-70.

[14] D. A. Hoffman and R. Osserman, The geometry of the generalized Gauss map, Memoirs of AMS, Number 236.

[15] T. Ilmanen, Singularity of mean curvature flow of surfaces, preprint.

[16] A. Neves, Singularities of Lagrangian mean curvature flow: monotone case, preprint.

[17] A. Neves, Singularities of Lagrangian mean curvature flow: zero-Maslov class case, Invent. Math., 168 (3) (2007), 449-484.

[18] R. Osserman, Geometry V, minimal surfaces, Springer, 1997.

[19] K. Smoczyk, Der Lagrangesche mittlere Kruemmungsfluss. Univ. Leipzig (Habil.-Schr.), 102 S. 2000.

[20] K. Smoczyk, Harnack inequality for the Lagrangian mean curvature flow, Calc. Var. PDE, 8 (1999), 247-258. 
[21] K. Smoczyk, Angle theorems for the Lagrangian mean curvature flow, Math. Z., 240 (2002), 849-883.

[22] R. Thomas and S.T. Yau, Special Lagrangians, stable bundles and mean curvature flow, math.DG/0104197 (2001).

[23] M.-T. Wang, Mean curvature flow of surfaces in Einstein four manifolds, J. Diff. Geom., 57 (2001), 301-338.

[24] B. White, The nature of singularities in mean curvature flow of mean-convex sets, J. Amer. Math. Soc., 16 (2003), 123-138.

[25] B. White, A local regularity theorem for mean curvature flow, Ann. Math. 161(2005), 14871519.

Math. Group, The abdus salam ICTP, Trieste 34100, Italy

E-mail address: xhan@ictp.it

Math. Group, The abdus salam ICTP, Trieste 34100, Italy, and Academy of Mathematics And Systems Sciences, Chinese Academy of Sciences, Beijing 100080, P. R. of ChINA.

E-mail address: jyli@ictp.it 